\title{
Three-dimensional numerical study of a fluid-kinetic model for respiratory aerosols with variable size and temperature
}

\author{
Laurent Boudin and David Michel \\ Sorbonne Université and Université de Paris, CNRS, \\ Laboratoire Jacques-Louis Lions (LJLL), \\ 4 place Jussieu \\ 75005 Paris, France \\ laurent.boudin@sorbonne-universite.fr; \\ david.michel.1@sorbonne-universite.fr
}

October 25, 2020

\begin{abstract}
In this paper, we extend to the three-dimensional case the numerical study previously performed in a two-dimensional framework for a complex coupled fluid-kinetic model describing respiratory aerosols. The specificity of this model lies in the fact that it takes into account the airways humidity and the resulting hygroscopic effects on the aerosol droplets, namely their size variation. The air is described through a system of partial differential equations: the incompressible Navier-Stokes equations for the air velocity, convection-diffusion equations on its temperature and water vapor mass fraction. The aerosol distribution function solves a Vlasov-type equation and depends on the standard kinetic variables, but also on radius and temperature variables. After discussing again the implementation strategy, we perform numerical experiments, mainly in a branched structure looking like the trachea and the first lung generation. This allows to present various statistics on the aerosol behavior in terms of particle deposition, temperature and size variation of the droplets. We observe that the outcome appears coherent with the two-dimensional case. Finally, we discuss several assumptions which may lead to model simplifications, such as the fact that the water vapor mass fraction in the air may be considered to be constant throughout the branched structure in standard breathing conditions.
\end{abstract}




\section{Introduction}

The numerical simulation of an aerosol flow, and in particular the deposition phenomenon, in the human airways is of critical importance to investigate aerosol therapy, one of the main treatments of chronic obstructive pulmonary diseases (COPD). The key issue to this approach is to deliver a drug to the obstructed region of the airways as efficiently as possible.

For the purposes of this technique, the medication is mixed with an excipient and water through a nebulizer system to create a large number of droplets, the aerosol. Since the airways are a humid medium, the question of aerosol hygroscopic properties is natural to tackle, see [12, 13, 14. In particular, the aerosol droplets may exchange aqueous matter with the air loaded with water vapour in the lung. And that may imply some size variation of the droplets.

Two possible ideas for the modelling of aerosol evolution in the air are to either consider the particles individually or as a proper fluid mixed to the air. The former consists in following individual properties of the droplets forming the aerosol, as in [15, 18], and results in a computational challenge because of the very large number of physical particles under consideration. For example, for a publicly distributed nebulizer, 4] calculates that $10^{10}$ particles can be injected in the airways in one minute. The latter point of view gives rise to so-called two phase models, e. g. [1, 7, which may not be able to provide accurate information on the particle deposition areas.

In this context, fluid-kinetic models appear well-suited to provide a good description, the main reason being that the volume occupied by the aerosol remains negligible with respect to the airways volume. We also assume that the particles do not interact with one another and that their action on the air can be neglected. Therefore, following the nomenclature introduced in [16], we consider a very thin spray of particles, which was partially studied in [4].

Let us now discuss how to handle the specific hygroscopic phenomena. After the first major attempts by [12, 13], [3] presents a set of partial differential equations which describes them as local effects. In this framework, a distribution function solving a Vlasov-type equation characterizes the aerosol. This function depends on time, position, velocity, size, and temperature. The fluid unknowns are, on the one hand the velocity and pressure, and on the other hand the temperature and the water vapor mass fraction. The former classically satisfy the incompressible Navier-Stokes equations, which do not involve the aerosol under the very thin spray assumption. The latter are both subject to convection and diffusion phenomena and therefore satisfy convection-diffusion equations. The thermal and matter exchanges between 
the air and the aerosol appear as forcing terms in these equations. Therefore, the resulting system is strongly coupled. As opposed to [12, 14, 3] does not take into account other effects, such as turbulence.

The mathematical aspects of fluid-kinetic models has been investigated intensively for the past decades, e. g. in [8, 9, 17]. In the context of respiratory aerosols, see [2, 5] for example. More recently, [6] proves the existence of global weak solutions for a more complex system than the one under review in this article, in a time-dependent domain.

A numerical method to tackle this model is presented in the dimension two in [3]. Our first goal is to extend this approach to a three-dimensional framework. Then we shall focus on possible simplifications that could alleviate the computational effort while providing accurate results.

This article is divided into three main parts. In Section 2 we recall the model first presented in [3], taking into account a simplification related to the small size of the aerosol droplets. We then provide in Section 3 a few details on the numerical scheme and its implementation in three dimensions. Finally, Section 4 is dedicated to numerical experiments for the complete model and under several assumptions, which provide some leads on new relevant simplifications of the model.

\section{Presentation of the model}

As previously stated, this section recalls the model presented in [3] to describe the behavior of an aerosol in the respiratory system and, more precisely, we focus on the trachea and the first two branches of the airways. We denote by $\Omega \subset \mathbb{R}^{3}$ this open bounded set. Its boundary $\Gamma=\partial \Omega$ is divided into three subsets, the wall $\Gamma^{\text {wall }}$, the inlet $\Gamma^{\text {in }}$ and the outlet $\Gamma^{\text {out }}$, suggesting that we focus on the inhaling part of the respiration mechanism.

We consider the aerosol as a dispersed phase and use a kinetic approach to model it. Therefore, we introduce the aerosol distribution function $f$. It depends on time $t \geq 0$, position $x \in \Omega$, velocity $v \in \mathbb{R}^{3}$, size $r>0$ and temperature $T>0$, and represents the density of the particles in the phase space $\Omega \times \mathbb{R}^{3} \times \mathbb{R}_{+}^{*} \times \mathbb{R}_{+}^{*}$. The dependence of $f$ on the size and temperature variables is a novelty introduced in [3] which stems from taking into account the matter and thermal exchanges between the aerosol and the surrounding air. Moreover, we assume that the droplets remain spherical, implying that $r$ can be chosen as the radius of a particle.

In order to describe the size variation phenomenon, we consider a particle of radius $r>0$. Schematically, we suppose that each droplet is divided into 
three layers: the active product, the excipient, and the water. The inner ball with the active product has the radius $r_{\text {drug }}>0$. The corona with the excipient has an outer radius $r_{\mathrm{ex}} \geq r_{\mathrm{drug}}$. Finally, the rest of the ball is composed of water and has a radius $r \geq r_{\mathrm{ex}}$. We assume that $r_{\mathrm{drug}}$ and $r_{\mathrm{ex}}$ remain constant and that the only size variation comes from the condensation or evaporation of water at the surface of the particles. If we denote by $\rho_{\mathrm{w}}$, $\rho_{\text {drug }}$ and $\rho_{\text {ex }}$ the mass densities of water, drug and excipient, the drug mass in a particle is $\frac{4}{3} \pi r_{\mathrm{drug}}^{3} \rho_{\mathrm{drug}}$, the excipient mass is $\frac{4}{3} \pi\left(r_{\mathrm{ex}}^{3}-r_{\mathrm{drug}}^{3}\right) \rho_{\mathrm{ex}}$ and the water mass is $\frac{4}{3} \pi\left(r^{3}-r_{\mathrm{ex}}^{3}\right) \rho_{\mathrm{w}}$. We assume all the mass densities to be constant, so that the mass and mass density of a particle only depend on $r$ and are given by

$$
\begin{aligned}
& m(r)=\frac{4}{3} \pi\left[r_{\mathrm{drug}}^{3} \rho_{\mathrm{drug}}+\left(r_{\mathrm{ex}}^{3}-r_{\mathrm{drug}}^{3}\right) \rho_{\mathrm{ex}}+\left(r^{3}-r_{\mathrm{ex}}^{3}\right) \rho_{\mathrm{w}}\right], \\
& \rho_{\mathrm{d}}(r)=\frac{1}{r^{3}}\left[r_{\mathrm{drug}}^{3} \rho_{\mathrm{drug}}+\left(r_{\mathrm{ex}}^{3}-r_{\mathrm{drug}}^{3}\right) \rho_{\mathrm{ex}}+\left(r^{3}-r_{\mathrm{ex}}^{3}\right) \rho_{\mathrm{w}}\right] .
\end{aligned}
$$

The aerosol moves inside the air, assumed to be a Newtonian and incompressible fluid. Hence, the motion of the air can be described by its velocity $u$ and pressure $p$, both depending on time $t \geq 0$ and position $x \in \Omega$. We also need to introduce the water vapor mass fraction in the air $Y_{\mathrm{v} \text {,air }}$ and the air temperature $T_{\text {air }}$ to account for the aforementioned water vapor exchanges with the aerosol. These quantities $Y_{\mathrm{v} \text {, air }}$ and $T_{\text {air }}$ also depend on time $t \geq 0$ and position $x \in \Omega$.

We can now write the equations governing the model. The density satisfies the Vlasov-type equation

$$
\left.\partial_{t} f+v \cdot \nabla_{x} f+\operatorname{div}_{v}[\alpha(u-v)+g) f\right]+\partial_{r}(a f)+\partial_{T}(b f)=0,
$$

where $g$ is the gravitational field, $\alpha(u-v)$ is the drag acceleration related to the motion of the air, and $a$ and $b$ respectively describe the radius and temperature variations. We have

$$
\alpha(r)=\frac{6 \pi \eta r}{m(r)}
$$

where $\eta$ is the constant air dynamic viscosity, and

$$
\begin{gathered}
a\left(r, T, Y_{\mathrm{v}, \text { air }}(t, x)\right)=-\frac{N_{\mathrm{d}}\left(r, T, Y_{\mathrm{v}, \text { air }}(t, x)\right)}{\rho_{\mathrm{w}}}, \\
b\left(r, T, Y_{\mathrm{v}, \text { air }}(t, x), T_{\mathrm{air}}(t, x)\right)=\frac{3}{\rho_{\mathrm{d}}(r) c_{P_{\mathrm{d}} r}}\left(-Q_{\mathrm{d}}\left(r, T, T_{\text {air }}(t, x)\right.\right. \\
\left.\left.-L_{\mathrm{v}} N_{\mathrm{d}}\left(r, T, Y_{\mathrm{v}, \text { air }}(t, x)\right)\right)\right),
\end{gathered}
$$


where $N_{\mathrm{d}}$ denotes the water mass flux at the droplet surface, $C_{P_{\mathrm{d}}}$ the specific heat of the aerosol, $Q_{\mathrm{d}}$ the convective flux between the air the the particle, and $L_{\mathrm{v}}$ the latent heat of water vaporization. We have

$$
Q_{\mathrm{d}}\left(r, T, T_{\mathrm{air}}(t, x)\right)=\frac{\mathrm{Nu} \kappa_{\mathrm{air}} C_{T}}{2 r}\left(T-T_{\text {air }}(t, x)\right),
$$

where $\mathrm{Nu}$ is the droplet Nusselt number in the air, $\kappa_{\text {air }}$ the thermal conductivity of the air as a gaseous mixture, and $C_{T}$ the Knudsen correlation for non-continuum effects. All these parameters are assumed to be constant. The expression for $N_{\mathrm{d}}$ is more complex and we first need to introduce several physical quantities. We begin by defining the water vapor saturation pressure, as a function of $T$, that can be expressed in the cgs unit system by

$$
P_{\mathrm{v}, \mathrm{sat}}(T)=10 \exp \left(23.196-\frac{3816.44}{T-46.13}\right) .
$$

Then, we account for the Kelvin effect on the droplet surface concentration of water vapor with

$$
K(r, T)=\exp \left(\frac{2 \sigma}{r \rho_{\mathrm{d}}(r) R_{\mathrm{v}} T}\right),
$$

where $R_{\mathrm{v}}$ is the gas constant of water vapor and $\sigma$ is the droplet surface tension, assumed to be constant. We also need the water activity coefficient

$$
S(r)=\frac{\frac{\rho_{\mathrm{w}}\left(r^{3}-r_{\mathrm{ex}}^{3}\right)}{M_{\mathrm{w}}}}{\frac{\rho_{\mathrm{w}}\left(r^{3}-r_{\mathrm{ex}}^{3}\right)}{M_{\mathrm{w}}}+i_{\mathrm{drug}} \frac{\rho_{\mathrm{drug}} r_{\mathrm{drug}}^{3}}{M_{\mathrm{drug}}}+i_{\mathrm{ex}} \frac{\rho_{\mathrm{ex}}\left(r_{\mathrm{ex}}^{3}-r_{\mathrm{drug}}^{3}\right)}{M_{\mathrm{ex}}}},
$$

where $M_{\mathrm{w}}, M_{\mathrm{drug}}$ and $M_{\mathrm{ex}}$ are the molar masses of water, drug and excipient, respectively, and $i_{\mathrm{drug}}$ and $i_{\mathrm{ex}}$ are the Van't Hoff factors of the drug and the excipient. We can now define the mass fraction of water vapor at the droplet surface

$$
Y_{\mathrm{v}, \text { surf }}(r, T)=\frac{S(r) K(r, T) P_{\mathrm{v}, \mathrm{sat}}(T)}{\rho_{\mathrm{d}}(r) R_{\mathrm{v}} T} .
$$

The water mass flux is then given by

$$
N_{\mathrm{d}}\left(r, T, Y_{\mathrm{v}, \text { air }}(t, x)\right)=\rho_{\text {air }} \frac{\operatorname{Sh} D_{\mathrm{v}}\left(T_{\text {air }}\right) C_{m}}{2 r} \frac{Y_{\mathrm{v}, \text { surf }}(r, T)-Y_{\mathrm{v}, \text { air }}(t, x)}{1-Y_{\mathrm{v}, \text { surf }}(r, T)},
$$


where $\rho_{\text {air }}$ is the air mass density, Sh the Sherwood number, $C_{m}$ the mass Knudsen number correction, and $D_{\mathrm{v}}$ is the binary diffusion coefficient of water vapor in the air and is given, in the cgs unit system, by

$$
D_{\mathrm{v}}\left(T_{\text {air }}(t, x)\right)=0.216\left(\frac{T_{\text {air }}(t, x)}{273.15}\right)^{1.8} .
$$

Equation (3) is supplemented by the deposition condition on $\Gamma^{\text {wall }} \times \mathbb{R}^{3} \times$ $\mathbb{R}_{+}^{*} \times \mathbb{R}_{+}^{*}$

$$
f(t, x, v, r, T)=0 \quad \text { if } v \cdot n \leq 0,
$$

and the initial condition on $\Omega \times \mathbb{R}^{3} \times \mathbb{R}_{+}^{*} \times \mathbb{R}_{+}^{*}$

$$
f(0, x, v, r, T)=f^{0},
$$

where $f^{0}: \Omega \times \mathbb{R}^{3} \times \mathbb{R}_{+}^{*} \times \mathbb{R}_{+}^{*} \rightarrow \mathbb{R}_{+}$is a given function.

As stated before, the air is first described through its velocity $u(t, x) \in \mathbb{R}^{3}$ and its pressure $p(t, x) \in \mathbb{R}$, which solve the incompressible Navier-Stokes equations

$$
\begin{aligned}
\rho_{\text {air }}\left(\partial_{t} u+\left(u \cdot \nabla_{x}\right) u\right)-\eta \Delta_{x} u+\nabla_{x} p & =0, \\
\operatorname{div}_{x} u & =0 .
\end{aligned}
$$

Following [3, 4, we choose to neglect the aerosol retroaction on the air since we deal with small-sized particles. Equations (16)-(17) are supplemented with the following boundary and initial conditions:

$$
\left\{\begin{array}{rlrl}
u & =u^{\text {in }} & & \text { on } \mathbb{R}_{+} \times \Gamma^{\text {in }} \\
u=0 & & \text { on } \mathbb{R}_{+} \times \Gamma^{\text {wall }} \\
\sigma(u, p) \cdot n & =0 & \text { on } \mathbb{R}_{+} \times \Gamma^{\text {out }} \\
& u(0, \cdot)=u^{0}
\end{array}\right.
$$

where $\sigma(u, p)=\nabla_{x} u+\left(\nabla_{x} u\right)^{\top}-p \operatorname{Id}$ is the stress tensor, $n$ is the outgoing normal vector from $\Gamma$, and $u^{\text {in }}: \mathbb{R}_{+} \times \Gamma^{\text {in }} \rightarrow \mathbb{R}^{3}, u^{0}: \Omega \rightarrow \mathbb{R}^{3}$ are given.

The water vapor mass fraction $Y_{\mathrm{v} \text {,air }}$ satisfies the following convectiondiffusion equation on $\mathbb{R}_{+} \times \Omega$

$$
\rho_{\text {air }}\left(\partial_{t} Y_{\mathrm{v}, \text { air }}+u \cdot \nabla_{x} Y_{\mathrm{v}, \text { air }}\right)-\operatorname{div}_{x}\left(D_{\mathrm{v}}\left(T_{\text {air }}\right) \nabla_{x} Y_{\mathrm{v}, \text { air }}\right)=S_{Y},
$$

where $S_{Y}$ accounts for the water mass exchanges between the air and the particles and is given by

$$
S_{Y}(t, x)=\rho_{\mathrm{w}} \int_{\mathbb{R}^{3} \times \mathbb{R}_{+}^{*} \times \mathbb{R}_{+}^{*}} 4 \pi r^{2} N_{\mathrm{d}}\left(r, T, Y_{\mathrm{v}, \text { air }}(t, x)\right) f(t, x, v, r, T) \mathrm{d} v \mathrm{~d} r \mathrm{~d} T .
$$


Equation 20 is supplemented with the boundary conditions

$$
\left\{\begin{aligned}
Y_{\mathrm{v}, \text { air }} & =Y_{\mathrm{v}, \text { air }}^{\text {in }} & & \text { on } \mathbb{R}_{+} \times \Gamma^{\text {in }} \\
Y_{\mathrm{v}, \text { air }} & =Y_{\mathrm{v}, \text { wall }} & & \text { on } \mathbb{R}_{+} \times \Gamma^{\text {wall }} \\
\nabla_{x} Y_{\mathrm{v}, \text { air }} \cdot n & =0 & & \text { on } \mathbb{R}_{+} \times \Gamma^{\text {out }}
\end{aligned}\right.
$$

and the initial condition on $\Omega$

$$
Y_{\mathrm{v}, \text { air }}(0, \cdot)=Y_{\mathrm{v}, \text { air }}^{0}
$$

where the constants $Y_{\mathrm{v}, \text { air }}^{\text {in }}, Y_{\mathrm{v} \text {,wall }}, Y_{\mathrm{v}, \text { air }}^{0} \in(0,1)$ are given.

Similarly, the air temperature $T_{\text {air }}$ satisfies on $\mathbb{R}_{+} \times \Omega$

$$
\rho_{\text {air }}\left(\partial_{t} T_{\text {air }}+u \cdot \nabla_{x} T_{\text {air }}\right)-\kappa_{\text {air }} \Delta_{x} T_{\text {air }}=S_{T},
$$

where $S_{T}$ accounts for the heat transfer between the air and the aerosol and is given by

$$
S_{T}(t, x)=\int_{\mathbb{R}^{3} \times \mathbb{R}_{+}^{*} \times \mathbb{R}_{+}^{*}} 4 \pi r^{2} Q_{\mathrm{d}}\left(r, T, T_{\text {air }}(t, x)\right) f(t, x, v, r, T) \mathrm{d} v \mathrm{~d} r \mathrm{~d} T .
$$

Equation (24) is supplemented with the boundary conditions:

$$
\left\{\begin{aligned}
T_{\text {air }} & =T_{\text {air }}^{\text {in }} & & \text { on } \mathbb{R}_{+} \times \Gamma^{\text {in }} \\
T_{\text {air }} & =T_{\text {wall }} & & \text { on } \mathbb{R}_{+} \times \Gamma^{\text {wall }} \\
\nabla_{x} T_{\text {air }} \cdot n & =0 & & \text { on } \mathbb{R}_{+} \times \Gamma^{\text {out }}
\end{aligned}\right.
$$

and the initial condition on $\Omega$

$$
T_{\text {air }}(0, \cdot)=T_{\text {air }}^{0}
$$

where the constants $T_{\text {air }}^{\text {in }}, T_{\text {wall }}, T_{\text {air }}^{0} \in \mathbb{R}_{+}^{*}$ are given.

Equations (3)-(27) form a strongly coupled system. We aim to solve this system numerically, provide quantitative results both for the air and the aerosol, as well as to discuss possible simplifications in the full threedimensional model.

\section{Numerical approach}

We implement the same numerical scheme as in [3] but in three dimensions. The reader is invited to refer to this article for more details about the scheme itself. In the following, we only recall the working assumptions 


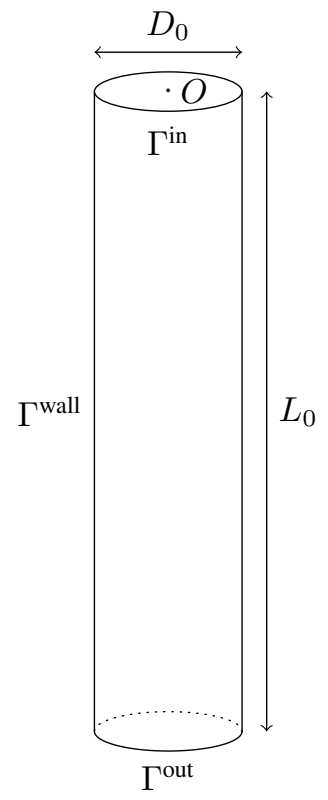

(a)

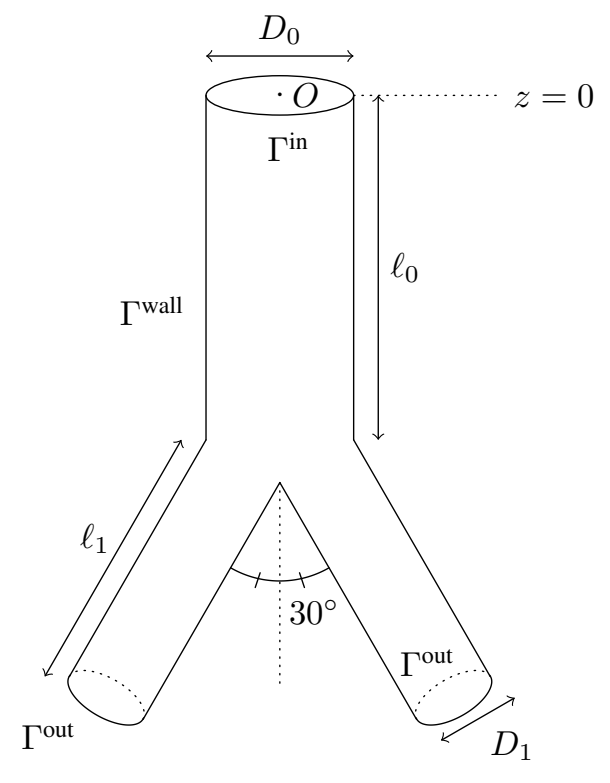

(b)

Figure 1: Schematic view of (a) the cylindrical domain, (b) the branched structure.

and provide some remarks on the implementation. All the computations are performed with FreeFem++ [10]

Our computations are performed on two distinct domains. The first one is a cylinder of diameter $D_{0}=0.8 \mathrm{~cm}$ and length $L_{0}=9.4 \mathrm{~cm}$, see Figure 1 a. The second one is a branched structure modelling an idealized trachea and the first generation of the airways. The length of the trachea is $\ell_{0}=2.9 \mathrm{~cm}$ and its diameter is $D_{0}$. Both bronchi form an angle of $30^{\circ}$ with the vertical axis, their length is set to $\ell_{1}=1.8 \mathrm{~cm}$, and their diameter is $D_{1}=0.6 \mathrm{~cm}$, see Figure $1 \mathrm{~b}$. In both cases, the center of the boundary $\Gamma^{\text {in }}$ is the origin of the space coordinate system.

The cylindrical domain mostly serves as a tool to validate our threedimensional code. Since there is no aerosol retroaction term in (16), a Poiseuille profile at the inlet leads to a stationary Poiseuille flow solving the

\footnotetext{
${ }^{1}$ Note that we had to use version 3.43 as opposed to version 4.4 .2 which is currently available because the function convect did not seem to properly behave in three dimensions.
} 
Navier-Stokes equations in $\Omega$. We also checked that if the initial distribution of the particles has a cylindrical symmetry, then it is preserved through time. Finally, we use the cylindrical domain in Section 4 to test model simplifications in a simpler geometrical and computational setting than the branched structure.

As stated above, the aerosol retroaction term on the fluid in (16) is set to 0, the Navier-Stokes equations (16)-(17) are uncoupled from the other ones and can be solved independently. This hypothesis is of significant importance as we are led to execute lengthy three-dimensional calculations only once. We therefore solve (16)- 17) with initial condition $u^{0}=0$ and, at the domain inlet, a boundary condition $u^{\text {in }}$ following a Poiseuille law, oriented vertically downwards and its modulus given, for $(x, y, 0) \in \Gamma^{\text {in }}$, by

$$
\left|u^{\text {in }}(x, y, 0)\right|=u_{0}\left(1-\frac{x^{2}+y^{2}}{\left(D_{0} / 2\right)^{2}}\right)
$$

with $u_{0}=50.0 \mathrm{~cm} . \mathrm{s}^{-1}$. When we solve the equations, the velocity seems to reach a stationary state, at least in the domains we studied. We use this solution in the rest of the computations.

Next, we consider the following conditions on the air temperature:

$$
T_{\text {air }}^{0}=310 \mathrm{~K}, \quad T_{\text {air }}^{\text {in }}=297 \mathrm{~K}, \quad T_{\text {wall }}=310 \mathrm{~K} .
$$

Then, a comparison of the orders of magnitude of the coefficients in 20 shows that the leading term is the diffusion one and therefore $Y_{\mathrm{v} \text {,air }}$ should rapidly reach an equilibrium and the aerosol should not have any influence, which is confirmed by numerical simulations. For example, in the case of the branched structure, we have

$$
\begin{array}{cc}
\frac{\rho_{\text {air }}}{\Delta t} \sim 7 \times 10^{-1} \mathrm{~g} \cdot \mathrm{cm}^{-3} \cdot \mathrm{s}^{-1}, & \frac{\rho_{\text {air }}|u|}{\Delta x} \sim 5 \times 10^{-1} \mathrm{~g} \cdot \mathrm{cm}^{-3} \cdot \mathrm{s}^{-1}, \\
\frac{D_{\mathrm{v}}\left(T_{\text {air }}^{\text {in }}\right)}{(\Delta x)^{2}} \sim 11 \mathrm{~g} \cdot \mathrm{cm}^{-3} \cdot \mathrm{s}^{-1}, & \left|S_{Y}\right| \sim 5 \times 10^{-5} \mathrm{~g} \cdot \mathrm{cm}^{-3} \cdot \mathrm{s}^{-1},
\end{array}
$$

where $\Delta t$ is the time step and $\Delta x$ is the average diameter of a cell of the three-dimensional mesh. Moreover, it appears that the variation of $D_{\mathrm{v}}\left(T_{\text {air }}\right)$ as $T_{\text {air }}$ changes does not influence $Y_{\mathrm{v}, \text { air }}$. Therefore, we solve Equation (20) independently and $D_{\mathrm{v}}\left(T_{\text {air }}\right)$ is considered equal to $D_{\mathrm{v}}\left(T_{\text {air }}^{\text {in }}\right)$ and we set $Y_{\mathrm{v} \text {,air }}^{0}=Y_{\mathrm{v} \text {,air }}^{\text {in }}=1.81 \%$ and $Y_{\mathrm{v} \text {,air }}^{\text {wall }}=3.70 \%$. These remarks lead to a decreasing computation time, but less significant than in the case of the Navier-Stokes equations. 
Following Remark 7 from [4], we solve the Vlasov equation (3) thanks to a time subcycling strategy. At each time step, we solve as many systems of ODEs of the following form as there are numerical particles $p$, i. e.

$$
\begin{aligned}
& \dot{x}_{p}(t)=v_{p}(t) \\
& \dot{v}_{p}(t)=\alpha\left(r_{p}(t)\right)\left(u\left(t, x_{p}(t)\right)-v_{p}(t)\right)+g \\
& \dot{r}_{p}(t)=a\left(r_{p}(t), T_{p}(t), Y_{\mathrm{v}, \text { air }}\left(t, x_{p}(t)\right)\right), \\
& \dot{T}_{p}(t)=b\left(r_{p}(t), T_{p}(t), Y_{\mathrm{v}, \text { air }}\left(t, x_{p}(t)\right), T_{\mathrm{air}}\left(t, x_{p}(t)\right)\right) .
\end{aligned}
$$

Note that these ODEs for a given particle $p$ are independent from the ODEs for all the other ones and it is therefore natural to perform a parallel computation to solve them and thus greatly reduce the computation time. We must emphasize that the number of numerical particles remains very small with respect to the number of physical ones, which implies a slender computational cost.

Finally, in order to avoid needing a precise parametrization of the boundary when dealing with the deposition condition, i.e. to be able to simply load a mesh file and solve the equations and verify the deposition criterion below without more precise knowledge of the domain, we define a standard notion of distance to the considered subset $\Sigma$ of $\Gamma$ by solving

$$
\left\{\begin{array}{rll}
-\Delta_{x} g_{\Sigma}=1 & \text { on } \Omega \\
g_{\Sigma}=0 & \text { on } \Sigma \\
\nabla_{x} g_{\Sigma} \cdot n=0 & \text { on } \Gamma \backslash \Sigma
\end{array}\right.
$$

and setting

$$
\forall x \in \Omega, \quad d_{\Sigma}(x)= \begin{cases}\frac{g_{\Sigma}(x)}{\left\|\nabla_{x} g_{\Sigma}(x)\right\|} & \text { if } \frac{g_{\Sigma}(x)}{\nabla_{x} g_{\Sigma}(x)}<\eta \\ \eta & \text { else }\end{cases}
$$

and $d_{\Sigma}(x)=g_{\Sigma}(x)=0$ if $x \in \Sigma$, for some $\eta>0$. The functions $d_{\Sigma} \geq 0$ satisfy $d_{\Sigma}(x)=0 \Leftrightarrow x \in \Sigma$ and enable us to discriminate between particles near the boundary under consideration.

The deposition/exit test is therefore performed as follows at each time step of the subcycle solving the ODEs for the particles.

- If at some time step, the droplet is outside $\Omega$, we go back to the previous position (still inside the domain) and compare the distances of the particle to the wall, and both exits, and consider that the particle was deposited or exited accordingly. 
- If the droplet remains inside $\Omega$, we assume that it is deposited if both the following conditions are met:

$$
d_{\Gamma_{\text {wall }}}\left(x_{p}\right) \leq \alpha h_{\text {min }} \quad \text { and } \quad v_{p} \Delta t \leq \alpha h_{\text {min }},
$$

where $h_{\min }$ is the minimal diameter of a mesh cell and $\alpha>0$. Let us emphasize the fact that $\Delta t$ is the global time step, as opposed to the one used to the the ODEs. These conditions mean that if the particle is close enough to the wall and its velocity is small enough, then we consider the particle to be deposited. This is physically justified by the fact that under these assumptions, the mucus on the wall tends to force the adhesion of the particle to the wall, see [11.

Note that the definition of $d_{\Gamma \text { wall }}$ implies to take $\eta$ greater than $\alpha h_{\min }$ for the deposition test to make sense. Furthermore, we have investigated the influence of the choice of $\alpha$ for values between 0.5 and 2 and have found the deposition rate to be have a stable behavior. For instance, when a set of initial positions of the particles leads to 3 deposited numerical particles for $\alpha=0.5$, it leads to 19 deposited particles for $\alpha=2$. Finally, working under the initial conditions presented above, we find that the limiting factor for deposition is the distance to the wall rather than the velocity.

An intuitive choice would have been to say that a particle with radius $r_{p}$ at position $x_{p}$ is deposited if $d_{\Gamma^{w a l l}}\left(x_{p}\right) \leq r_{p}$. This is implemented in [3] and the numerical simulations are run on a very fine mesh. On the contrary, for the sake of simplicity, we choose to use a mesh sufficiently fine fluid-wise but not particle-wise. Therefore, we need to adapt the deposition condition as described above.

We also considered the following deposition criterion

$$
d_{\Gamma \text { wall }}\left(x_{p}\right) \leq \ell_{\text {lash }},
$$

where $\ell_{\text {lash }}=0.1 \mathrm{~cm}$ is the average length of a bronchial lash. It led to a deposition rate of $20 \%$, a different order of magnitude from the $8 \%$ obtained in [3] and was not further investigated but should be taken into account in further research.

\section{Numerical results}

\subsection{Full model}

As stated above, all the tests discussed below were investigated both in the cylindrical domain and the branched structure presented in Figure 1 
and led to the same kind of comments and conclusions. Therefore, we only present our results in the latter case.

Our experiment consists in releasing five waves of 100 numerical particles, each representing $10^{4}$ physical particles, between the times $\Delta t=$ $1.5345410^{-3} \mathrm{~s}$ and $101 \Delta t$. All the particles initially have the same velocity $v_{p}(0)=50 e_{z}\left(\right.$ in $\left.\mathrm{cm} . \mathrm{s}^{-1}\right)$, the same radius $r_{p}(0)=22.5 \mu \mathrm{m}$, and the same temperature $T_{p}(0)=T_{\text {air }}^{\text {in }}$. Their position is defined randomly in the disk of radius $D_{0} / 4$ centered on the origin and lying in the plane $z=0$.

As mentioned in the previous section, the air velocity $u$ and the water vapor mass fraction $Y_{\mathrm{v} \text {,air }}$ are assumed to be stationary. Figure 2 displays the profile of $|u|$ and $Y_{\mathrm{v} \text {,air }}$ in the cross-section $y=0$ of the bifurcation.

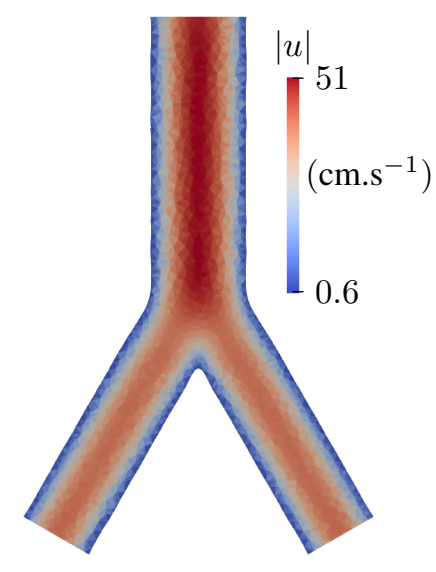

(a)

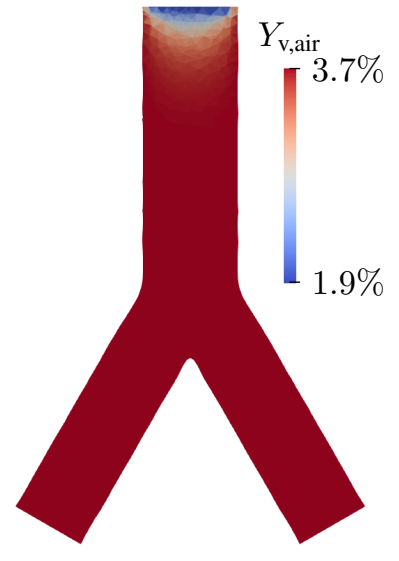

(b)

Figure 2: (a) Modulus of the velocity $|u|$ and (b) water vapor mass fraction $Y_{\mathrm{v}, \text { air }}$ in the cross-section $y=0$.

In Figure 3, we show the evolution of the particles in the domain. At first, in the trachea, the bundle of droplets assumes the shape of a paraboloid, which is consistent with the fact that the air flow is almost Poiseuille in this region.

The evolution of the air temperature as well as the particles is represented in Figure 4. Again, as expected, the temperature in the trachea evolves as if the air propagated as a Poiseuille flow, the cold wave assuming the shape of a parabola in the cross section $y=0$. We notice that the velocities of the droplets are a little bigger than that the fluid one, because of gravitational effects. 

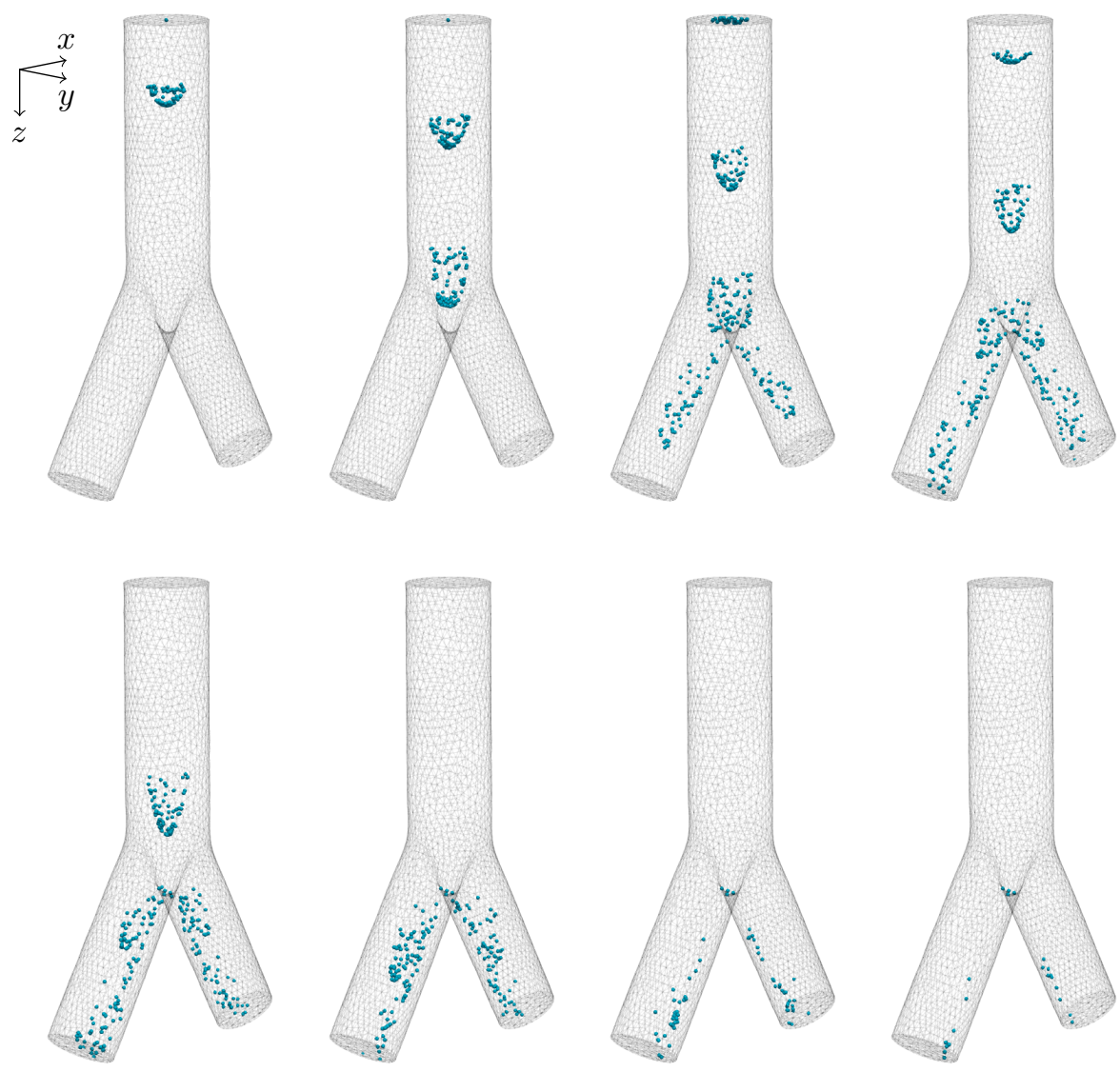

Figure 3: Dynamics of the particles at time $t=(10+25 i) \Delta t, 0 \leq i \leq 7$, i.e. between times $t_{0}=1.5345410^{-3} \mathrm{~s}$ and $t_{7}=2.838910^{-2} \mathrm{~s}$.

Figure 5 allows to see the local effects of the particles on the air temperature as it displays the temperature at a given time (a) with and (b) without the particles. As noticed in the two-dimensional case in [3], the droplets temperature is higher than the cold wave and they heat the air surrounding them as they go through the branched structure. This effect is not negligible as the air temperature around the particles can go up to about $302 \mathrm{~K}$.

Let us now present the results for the particles. The following plots are the results of one computation. Yet we find similar behaviors, from a statistical point of view, regardless of the initial uniform distribution of the particles in the disk of radius $D_{0} / 4$. In our test case, 5 particles are deposited, 239 exit the domain through the left branch (we use the patient's 

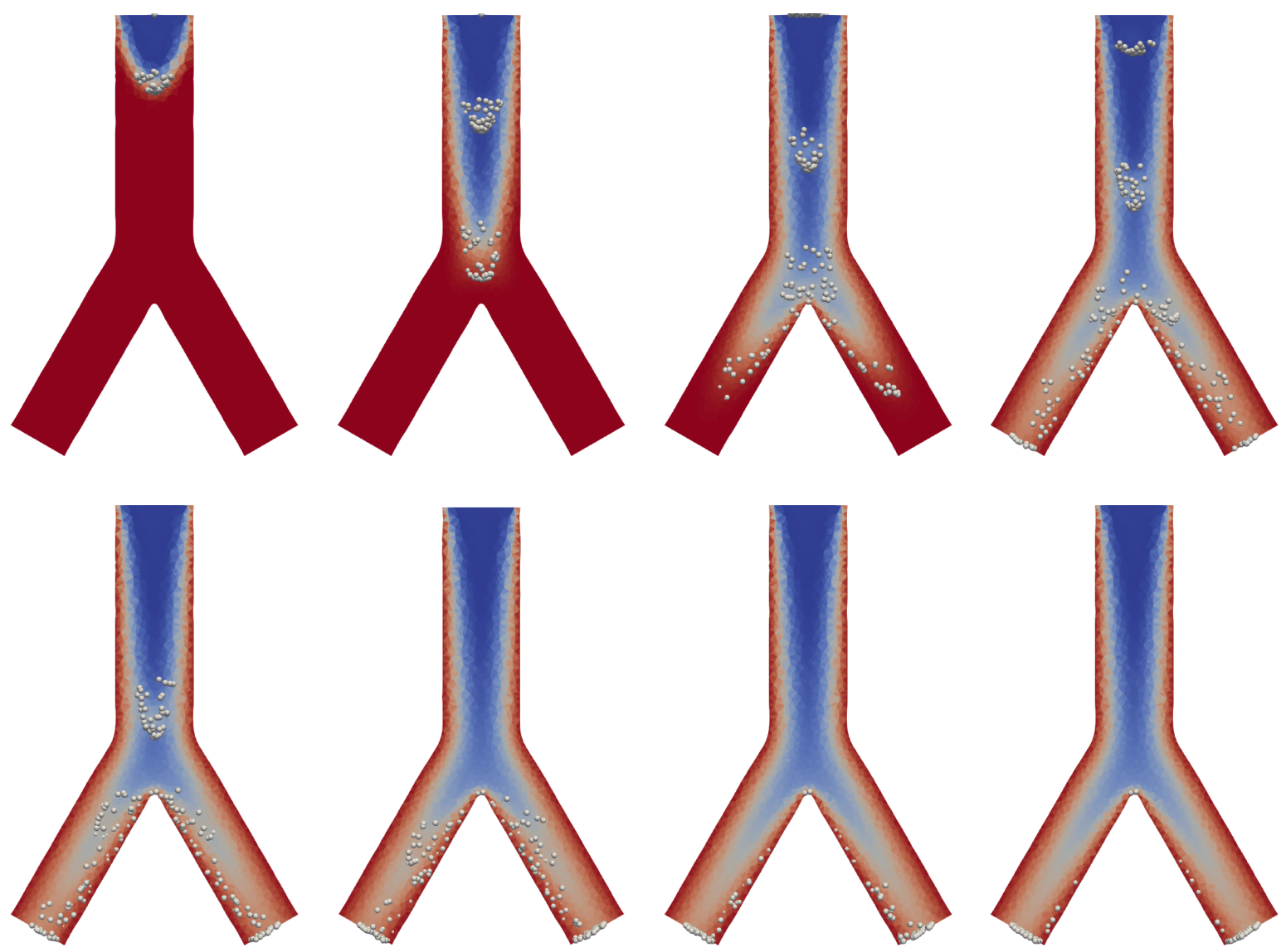

Figure 4: Dynamics of the particles and the air temperature, at times $t=$ $(10+25 \times i) \Delta t, 0 \leq i \leq 7$. The temperature is displayed on the domain $y=0$ while the particles with a positive $y$ coordinate are projected onto the plane $y=0$.

point of view) and 256 go through the right branch. Figure 6 displays their trajectories. We can see that only particles at the center of the inlet are deposited at the cusp of the branched structure and there is no deposition on the walls of the branches. The domain being asymmetrical in the case study of [3], we cannot draw much conclusions for the comparison of the results. Note nonetheless that the deposition of droplets, as expected, is lower for our three-dimensional domain. 


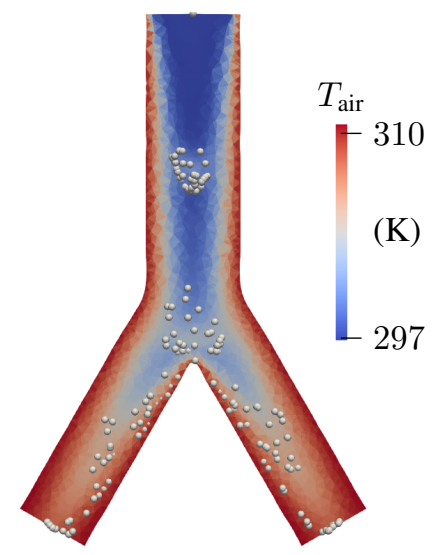

(a)

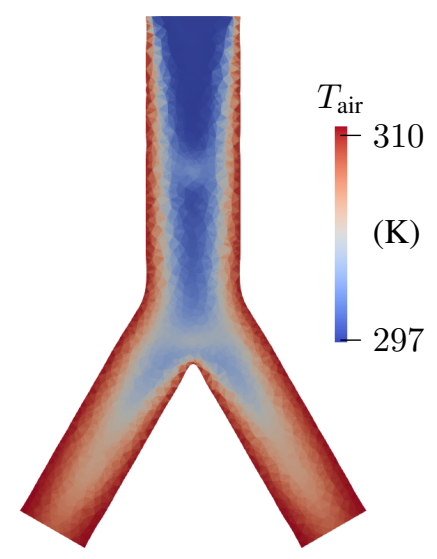

(b)

Figure 5: Local effects of the aerosol on the air temperature, at time $t=$ $0.12123 \mathrm{~s}$

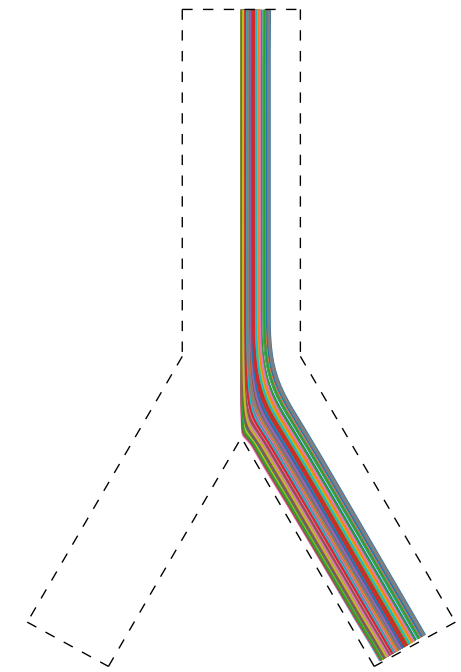

(a)

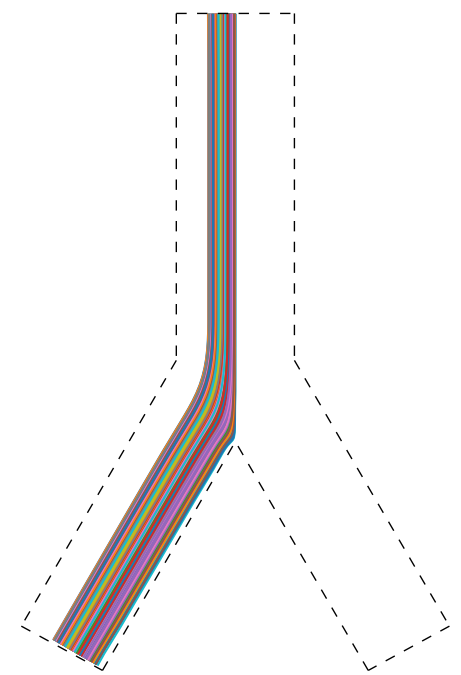

(b)

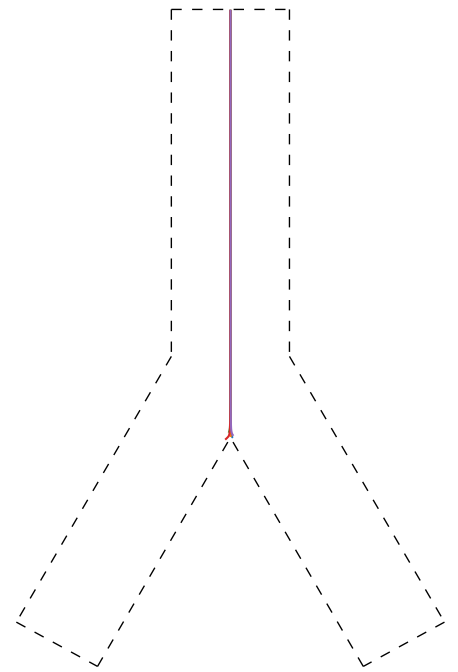

(c)

Figure 6: Particle trajectories in the reference case, the plane $y=0$ (a) towards the left branch, (b) towards the right branch, (c) until deposition. 


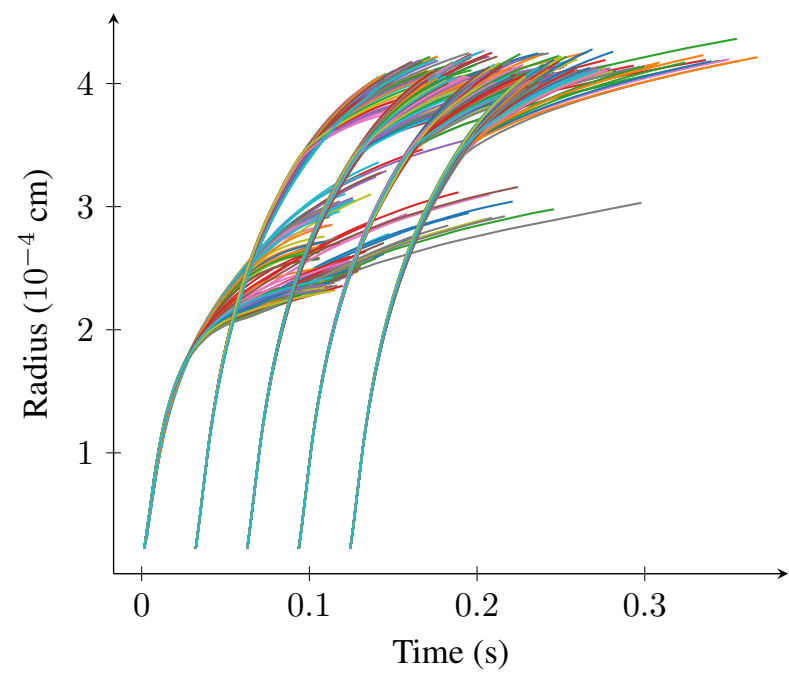

(a)

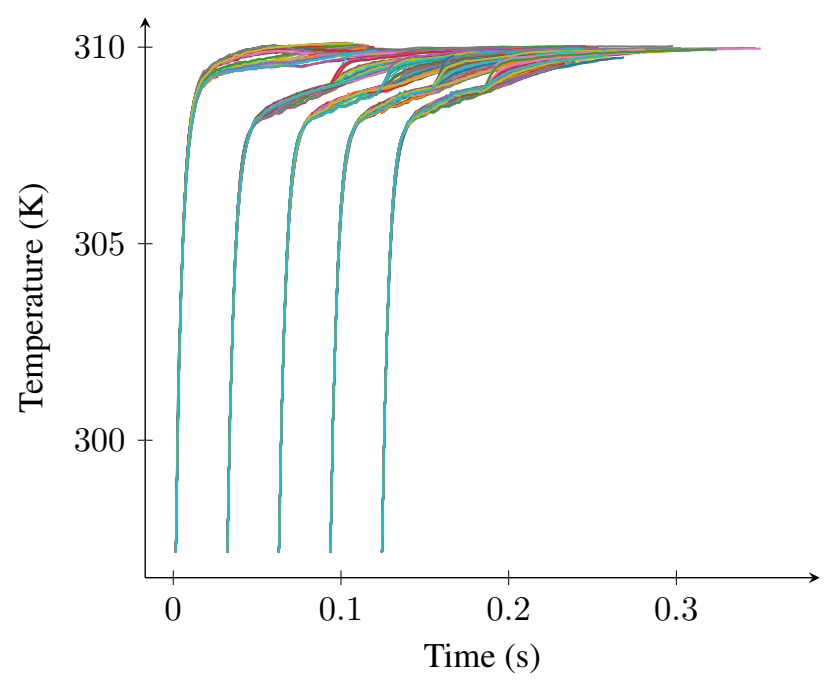

(b)

Figure 7: (a) Radius and (b) temperature evolution of the particles.

On Figure 7, we represent the evolution of the radius and temperature of the particles until their exit from the domain or their deposition. Similarly to what is described in two dimensions in [3], the first injection of particles behaves quite differently from the other four. The explanation lies in the fact that they encounter different air temperatures. Indeed, the first particles are transported through hot air, the cold wave being just behind them, while the other injections flow through cold air. Note that the entry of the particles into each branch coincides with the spreading of radii and temperatures among the injections.

Since we run computations with a random initial distribution of particles, we need to repeat the experiment 10 times and average to draw conclusions on the data we obtain. In Table 1 (Full Model column, Model B being described below), we provide the mean percentage of deposited particles and if particles passing through the left or right branch and the corresponding mean times, as well as the mean radius and temperature of the droplets after their exit or deposition. To determine the accuracy, we compute the standard deviation with two significant figures and approximate the mean value accordingly. For example, we find a standard deviation of $0.5210^{-4} \mathrm{~cm}$ for the radius of the deposited particles and, consequently, the mean value is 
given as $3.2610^{-4} \mathrm{~cm}$. Yet, for the temperature, we obtain a very low standard deviation of $0.082 \mathrm{~K}$, which is irrelevant with respect to the precision of the numerical scheme and we therefore only provide 3 significant figures for these data.

\begin{tabular}{|l|c|c|}
\hline Mean & Full Model & Model B \\
\hline Deposition rate & $1.62 \%$ & $1.62 \%$ \\
\hline Left exit rate & $49.1 \%$ & $49.1 \%$ \\
\hline Right exit rate & $49.28 \%$ & $49.28 \%$ \\
\hline Radius depos. $\left(10^{-4} \mathrm{~cm}\right)$ & 3.26 & 3.37 \\
\hline Radius left exit $\left(10^{-4} \mathrm{~cm}\right)$ & 3.82 & 3.91 \\
\hline Radius right exit $\left(10^{-4} \mathrm{~cm}\right)$ & 3.81 & 3.91 \\
\hline Temperature depos. $(\mathrm{K})$ & 310 & 310 \\
\hline Temperature left exit $(\mathrm{K})$ & 310 & 310 \\
\hline Temperature right exit $(\mathrm{K})$ & 310 & 310 \\
\hline Depos. time $(\mathrm{s})$ & 0.1423 & 0.1424 \\
\hline Left exit time $(\mathrm{s})$ & 0.1935 & 0.1934 \\
\hline Right exit time $(\mathrm{s})$ & 0.1938 & 0.1937 \\
\hline
\end{tabular}

Table 1: Statistics for the particles in the branched structure.

\subsection{Potential simplifications of the model}

Let us now consider simplifications that could be made to the model. Our main focus is the effect of such modifications on the eventual deposition and the location of the deposited particles, as well as the evolution of the size of the droplets. The first simplification is tested in both cylindrical and branched structure cases while the other ones are only tested in the cylinder.

\subsubsection{Can we assume that the water vapor mass fraction is con- stant in $\Omega$ ?}

As explained in the previous section, we consider the water vapor mass fraction $Y_{\mathrm{v} \text {,air }}$ to be constant in time. As we can see on Figure $2 \mathrm{~b}, Y_{\mathrm{v}}$,air does not vary much in the branched structure and only differs from $Y_{\text {wall }}$ close to the inlet. We now assume that $Y_{\mathrm{v}, \text { air }}=Y_{\text {wall }}$ is constant throughout the domain. The remaining equations are (3), (16)-17) and (24) and constitute Model B. For our experiments, we set the particles in the same initial conditions as above, and compare the results. We find that the global 
behavior of a droplet is not changed. If a particle exits (left or right) or is deposited with a space-dependent $Y_{\mathrm{v} \text {,air }}$, it does so with a constant $Y_{\mathrm{v} \text {,air }}$ too. Statistics on the mean radii, temperature and deposition/exit times are provided in Table 1 (Model B). In order to compare more accurately the differences between the models, Table 2 provides statistics on the relative errors that we compute for each particle of our 10 experiments. We do not provide the values for the temperature as the mean relative error is less than the precision that can be expected from the approximation scheme.

\begin{tabular}{|l|c|c|}
\hline & Mean relative error & Standard deviation \\
\hline Radius depos. & $3.5 \%$ & $1.9 \%$ \\
\hline Radius left exit & $2.7 \%$ & $1.3 \%$ \\
\hline Radius right exit & $2.7 \%$ & $1.3 \%$ \\
\hline Depos. time & $0.02 \%$ & $0.03 \%$ \\
\hline Left exit time & $0.05 \%$ & $0.09 \%$ \\
\hline Right exit time & $0.05 \%$ & $0.1 \%$ \\
\hline
\end{tabular}

Table 2: Statistics on the relative errors at end time between the Full Model and Model B.

\subsubsection{Can we further assume that water vapor mass fraction at the droplet surface is constant?}

As we explained above, we have systematically validated our code in the case of a cylindrical domain and the outcomes do not differ from the Reference case of the branched structure (except that there is no deposition in the cylinder). In particular, the assumption $Y_{\mathrm{v}, \text { air }}=Y_{\text {wall }}$ leads to results similar to those of the previous paragraph. To answer the question under review in the following, we only consider the case of a cylindrical domain.

As shown in Figure 8, the water vapor mass fraction at the droplet surface seems to quickly reach an equilibrium. Though, for the particles in the first injection, this value seems a little greater than for the other injections, we now consider that $Y_{\mathrm{v} \text {,surf }}$ is a constant. This simplifies the differential equation (30) satisfied by the radius as it becomes:

$$
\dot{r}=\frac{c D_{\mathrm{v}}\left(T_{\text {air }}\right)}{r}
$$

for some constant $c>0$. If $D_{\mathrm{v}}\left(T_{\text {air }}\right)$ were constant, the radii would then be expressed analytically thanks to a square root, which is in fact reminiscent 


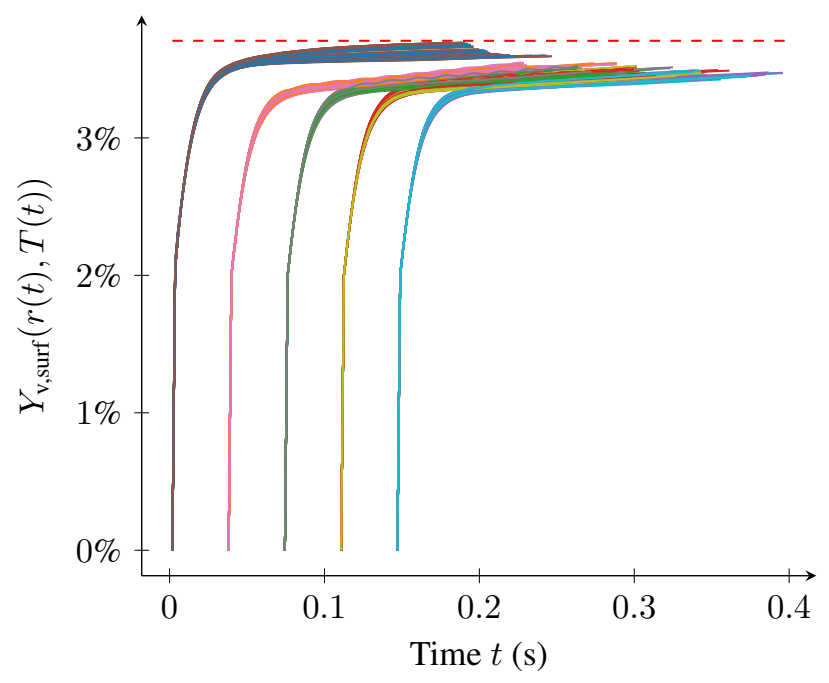

Figure 8: Water vapor mass fraction at the surface of the droplet in the cylinder. The dotted line represents the value $Y_{\mathrm{v}, \text { air }}=Y_{\text {wall }}$.

of the profiles of Figure $7 \mathrm{a}$. Yet the computations result in a droplet temperature behavior that is completely different from the reference case, as shown in Figure 9, and that does not seem physically relevant.

\subsubsection{Can we assume the air and particle temperatures to be constant?}

In [3], the authors show that considering the air and particle temperatures as constants results is $367 \%$ increase in the deposition rate and a $155 \%$ increase in the final mean radius. We have considered this hypothesis in the cylinder case and have found an increase of $30 \%$ in the final mean radius. Such an increase, in coherence with the two-dimensional results, is enough to discard the hypothesis and we do not investigate further the case of a branched structure.

Note that if we also consider $Y_{\mathrm{v} \text {,surf }}$ to be constant, equal to $3.5 \%$, then we obtain an increase of $18 \%$ in the final mean radius. 


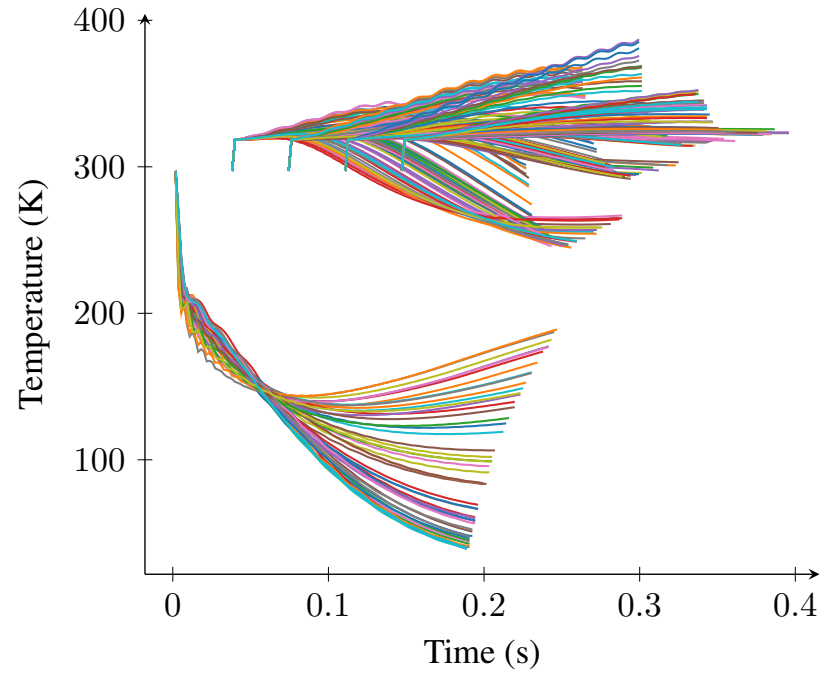

(a)

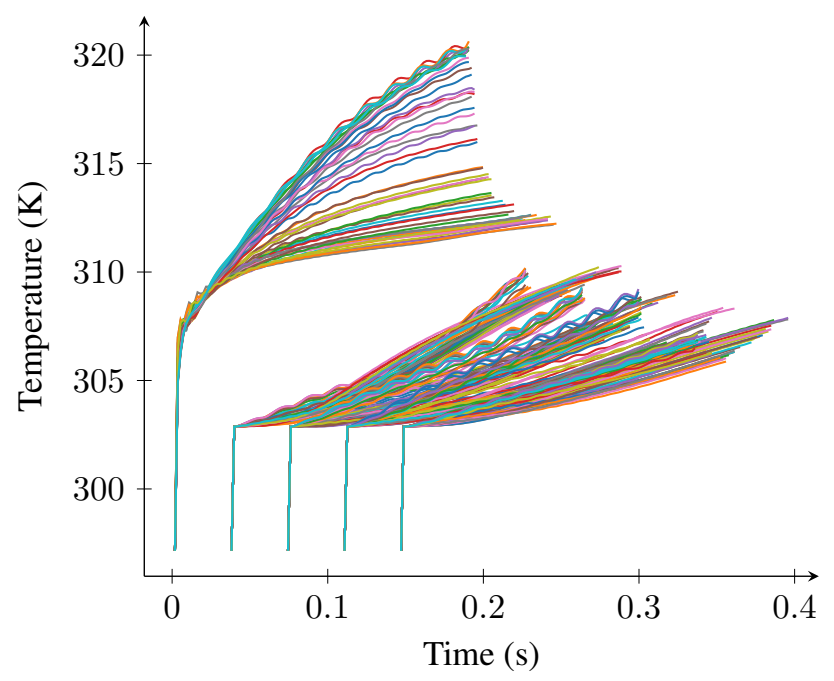

(b)

Figure 9: Temperature of the particles in the cylinder for $Y_{\mathrm{v} \text {,air }}=Y_{\text {wall }}$ and (a) $Y_{\mathrm{v}, \text { surf }}=0.03$ and (b) $Y_{\mathrm{v}, \text { surf }}=0.035$.

\section{Conclusions}

We can compare our results with those obtained in two dimensions in [3]. We find similar evolutions for the radius and temperature of the particles in the reference case. The particles flowing through the trachea also have a local influence on the air temperature, heating it by up to $1.6 \%$. Yet, the air temperature reaches a stationary state that does not depend on the fact that particles have been present in the domain.

As expected from the symmetry of the domain, the left and right exit rate are almost the same. The deposition phenomenon for small-sized particles seems to be mainly governed by the geometry of the domain and the initial position of the particles. An increase of around 3\% in the particle radius between the Full Model and Model B does not lead to a change in the deposition of the droplets. We cannot really comment on the deposition rate as the domain considered in 3] was more realistic and asymmetrical. To provide a meaningful comparison, we should compare our three-dimensional results to two-dimensional results in a symmetrical bifurcation. We could also create a three-dimensional mesh resembling the two-dimensional one 
from [3]. We should expect a lower deposition rate in dimension three since the particles have more space when they come close to the walls of the branch, see 4 .

When studying simplifications of the model, we find that the water vapor mass fraction in the air does not influence the behavior of the particles. Indeed, when we assume it is constant, it almost does not effect the temperature or trajectory of the droplets, and it induces a mean relative error on the radii of the deposited ones around 3.5\%, which can be neglected in this context. On the contrary, considering the water vapor mass fraction at the surface of the droplets to be constant leads to irrelevant results. Similarly if the water vapor mass fraction in the air and the particle and air temperatures are taken as constants, the changes on the radii are too significant for this simplification to be adopted.

As was already stated in [3], further investigations should focus on the effect of the excipient on the aerosol behavior, the effects of the geometry (since we only considered domains with a vertical main axis), or the influence of the initial radius on the deposition of the particles. The presence of mucus moving towards the inlet under the influence of the motion of the bronchial lashes should be modelled more precisely. Finally, taking into account the

variation of physical parameters or the presence of an obstruction in the airways would improve the modelling of respiratory diseases.

\section{Acknowledgements}

The authors want to thank Dr. Bérénice Grec and Prof. Sébastien Martin, from MAP5 of Université de Paris, for their input on the model and the computational aspects, as well as for their fruitful remarks on this work.

The first author wants to thank Dr. Laurent Vecellio, PST Director at Université de Tours and Prof. Stephan Ehrmann from CHRU Tours, as well as Prof. Céline Grandmont, from INRIA Paris and LJLL, for the various discussions they shared on the topic of aerosol size variation. 


\section{References}

[1] B. Asgharian, W. Hofmann, and R. Bergmann. Particle deposition in a multiple-path model of the human lung. Aerosol Sci. Tech., 34(4), pp. 332-339 (2001).

[2] L. Boudin, L. Desvillettes, C. Grandmont, and A. Moussa. Global existence of solutions for the coupled Vlasov and Navier-Stokes equations. Differential Integral Equations, 22(11-12), pp. 1247-1271 (2009).

[3] L. Boudin, C. Grandmont, B. Grec, S. Martin, A. Mecherbet, and F. Noël. Fluid-kinetic modelling for respiratory aerosols with variable size and temperature. ESAIM: ProcS., 67, pp. 100-119 (2020).

[4] L. Boudin, C. Grandmont, A. Lorz, and A. Moussa. Modelling and numerics for respiratory aerosols. Commun. Comput. Phys., 18(3), pp. 723-756 (2015).

[5] L. Boudin, C. Grandmont, and A. Moussa. Global existence of solutions to the incompressible Navier-Stokes-Vlasov equations in a timedependent domain. J. Differential Equations, 262(3), pp. 1317-1340 (2017).

[6] L. Boudin, D. Michel, and A. Moussa. Global existence of weak solutions to the incompressible Vlasov-Navier-Stokes system coupled to convection-diffusion equations. Math. Models Methods Appl. Sci., 30(8), pp. 1485-1515 (2020).

[7] A. Comerford, G. Bauer, and W. A. Wall. Nanoparticle transport in a realistic model of the tracheobronchial region. Int. J. Numer. Methods Biomed. Eng., 26(7), pp. 904-914 (2010).

[8] L. Desvillettes. Some aspects of the modeling at different scales of multiphase flows. Comput. Methods Appl. Mech. Engrg, 199(21-22), pp. 1265-1267 (2010).

[9] G. Dufour. Modélisation multi-fluide eulérienne pour les écoulements diphasiques à inclusions dispersées. $\mathrm{PhD}$ thesis, Université PaulSabatier Toulouse-III (2005).

[10] F. Hecht. New development in Freefem++. J. Numer. Math., 20(3-4), pp. 251-265 (2012). 
[11] C. S. Kim and M. A. Eldridge. Aerosol deposition in the airway model with excessive mucus secretions. J. Appl. Physiol., 59(6), pp. 1766-1772 (1985).

[12] P. W. Longest and M. Hindle. CFD simulations of enhanced condensational growth (ecg) applied to respiratory drug delivery with comparisons to in vitro data. J. Aerosol Sci., 41(8), pp. 805-520 (2010).

[13] P. W. Longest and M. Hindle. Numerical model to characterize the size increase of combination drug and hygroscopic excipient nanoparticle aerosols. Aerosol Sci. Tech., 45(7), pp. 884-899 (2011).

[14] P. W. Longest and C. Kleinstreuer. Computational models for simulating multicomponent aerosol drug and hygroscopic excipient nanoparticle aerosols. Aerosol Sci. Tech., 39(2), pp. 124-128 (2005).

[15] J. M. Oakes, A. L. Marsden, C. Grandmont, S. C. Shadden, C. Darquenne, and I. E. Vignon-Clémentel. Airflow and particle deposition simulations in health and emphysema: From in vivo to in silico animal experiments. Ann. Biomed. Eng., 42(4), pp. 899-914 (2014).

[16] P. J. O'Rourke. Collective drop effects on vaporizing liquid sprays. $\mathrm{PhD}$ thesis, Los Alamos National Laboratory (1981).

[17] F. A. Williams. Combustion theory. Benjamin Cummings, second edition, 1985.

[18] Z. Zhang, C. Kleinstreuer, and C. S. Kim. Airflow and nanoparticle deposition in a 16-generation tracheobronchial ariway model. Ann. Biomed. Eng., 36(12), pp. 2095-2110 (2008). 


\section{A Values of the physical constants}

All the values of the physical constants used in our numerical simulations are given in Table 3 and are the same as the ones collected in [3].

\begin{tabular}{|c|c|c|c|}
\hline Quantity & Symbol & Value & Unit (cgs) \\
\hline Gravitation & $|g|$ & 980 & $\mathrm{~cm} \cdot \mathrm{s}^{-2}$ \\
\hline Air mass density & $\rho_{\text {air }}$ & $1.1810^{-3}$ & g.cm ${ }^{-3}$ \\
\hline Air specific heat & $c_{P_{\text {air }}}$ & $1.0110^{7}$ & $\mathrm{~cm}^{2} \cdot \mathrm{s}^{-2} \cdot \mathrm{K}^{-1}$ \\
\hline Air thermal conductivity & $\kappa_{\text {air }}$ & $2.6010^{3}$ & erg.cm ${ }^{-1} \cdot \mathrm{s}^{-1} \cdot \mathrm{K}^{-1}$ \\
\hline Air dynamic viscosity & $\eta$ & $1.1810^{-4}$ & g.cm ${ }^{-1} \cdot \mathrm{s}^{-1}$ \\
\hline Water mass density & $\rho_{\mathrm{W}}$ & 0.997 & g.cm ${ }^{-3}$ \\
\hline Drug mass density & $\rho_{\text {drug }}$ & 1.34 & g.cm ${ }^{-3}$ \\
\hline Excipient mass density & $\rho_{\mathrm{ex}}$ & 2.17 & g.cm ${ }^{-3}$ \\
\hline Water molar mass & $M_{\mathrm{w}}$ & 18.0 & g.mol ${ }^{-1}$ \\
\hline Drug molar mass & $M_{\text {drug }}$ & 577 & g.mol ${ }^{-1}$ \\
\hline Excipient molar mass & $M_{\mathrm{ex}}$ & 58.4 & g. $\mathrm{mol}^{-1}$ \\
\hline Drug van't Hoff coefficient & $i_{\text {drug }}$ & 2.10 & - \\
\hline Excipient van't Hoff coefficient & $i_{\mathrm{ex}}$ & 2.10 & - \\
\hline Droplet specific heat & $c_{P_{\mathrm{d}}}$ & $4.1810^{7}$ & $\mathrm{~cm}^{2} \cdot \mathrm{s}^{-2} \cdot \mathrm{K}^{-1}$ \\
\hline Droplet mass Knudsen number correction & $C_{m}$ & 1.00 & - \\
\hline Droplet temperature Knudsen correlation & $C_{T}$ & 1.00 & - \\
\hline Droplet Nusselt number & $\mathrm{Nu}$ & 2.00 & - \\
\hline Droplet Sherwood number & Sh & 2.00 & - \\
\hline Water vaporization latent heat & $L_{\mathrm{v}}$ & $2.2610^{10}$ & $\mathrm{~cm}^{2} \cdot \mathrm{s}^{-2}$ \\
\hline Droplet surface tension & $\sigma$ & 72.0 & dyn.cm $^{-1}$ \\
\hline Water vapor specific gas constant & $R_{\mathrm{v}}$ & $4.6110^{6}$ & $\mathrm{~cm}^{2} \cdot \mathrm{s}^{-2} \cdot \mathrm{K}^{-1}$ \\
\hline
\end{tabular}

Table 3: Value of the physical constants. 